Vol 10, Issue 2, 2017

\title{
CONVENTIONAL AND RECENT DIAGNOSTIC AIDS IN TUBERCULOUS LYMPHADENITIS: A BRIEF OVERVIEW
}

\author{
SHAMIMUL HASAN ${ }^{1}$, SHAZINA SAEED ${ }^{2 *}$
}

${ }^{1}$ Department of Oral Medicine and Radiology, Faculty of Dentistry, Jamia Millia Islamia, New Delhi, India. ${ }^{2}$ Department of Amity Institute of Public Health, Amity University, Noida, Uttar Pradesh, India. Email: shs1091@gmail.com

\author{
Received: 09 November 2016, Revised and Accepted: 18 November 2016
}

\begin{abstract}
One of the most common form of extra-pulmonary tuberculosis (TB) is peripheral tuberculous lymphadenitis and accounts for $20-40 \%$ of the cases. Tuberculous lymphadenitis has a gender and age predilection (usually seen in young females). It is usually bilateral in presentation and is noncontagious. Recent upsurge in HIV coinfection has challenged the diagnosis and management of TB and of associated lymphadenopathy. In the endemic areas, tuberculous lymphadenitis remains an important and essential differential diagnosis in patients presenting with cervical swellings. A timely and accurate diagnosis is mandatory to overcome this public health threat. Interdisciplinary involvement of varied medical and dental professionals enhances the possibility of an effective and timely diagnosis of this condition.
\end{abstract}

Keywords: Cervical lymphadenopathy, Diagnosis, Management, Tuberculosis.

(c) 2017 The Authors. Published by Innovare Academic Sciences Pvt Ltd. This is an open access article under the CC BY license (http://creativecommons. org/licenses/by/4. 0/) DOI: http://dx.doi.org/10.22159/ajpcr.2017.v10i2.16102

\section{INTRODUCTION}

Tuberculosis (TB) is a multisystemic chronic infection caused by Mycobacterium TB and characterized by granuloma formation. The primary organ involved is the lungs, although disease other organs and systems may also be affected. $25 \%$ of the cases are attributable to extrapulmonary TB with $10-35 \%$ detected in the head and neck region $[1,2]$.

TB of the lymphatic system is the most common extra-pulmonary form of TB, second only to tuberculous pleurisy [3]. Cervical tuberculous lymphadenopathy (LD) accounts to the most frequent cause of persistent cervical lymphadenitis in the developing countries [4].

\section{CLINICAL FEATURES}

The predilection for younger age groups and female gender forms the distinguishing features for TB lymphadenitis [5]. The condition usually affects individuals in the age range of $20-40$ years [6]. The underprivileged state of women in rural Indian society may be a factor for unusual age and gender distribution [7].

Tuberculous lymphadenitis manifests as a slowly enlarging painless swelling of one or more lymph nodes of weeks to months interval. Systemic symptoms, i.e. fever, weight loss, fatigue and night sweats may occur in patients with extensive disease or coexisting disease. The initial presentation of TB lymphadenitis is firm, discrete, and mobile nodes with free overlying skin. Later, the nodes may become matted with inflamed overlying skin. Softening of the nodes with formation of abscesses and sinus tracts takes place in the advanced stages of the disease. Compression or invasion of the adjoining structures may occur in cases of unusually large nodes, complicating the course of the disease [8]

Jones and Campbell classification for tuberculous lymphadenitis - [9]

- Stage 1 - Reactive lymphadenitis - discrete, enlarged, mobile, Birm, nodes with features of nonspeciBic reactive hyperplasia

- Stage 2 - Periadenitis - Rubbery lymph nodes Bixed to adjoining tissues

- Stage 3 - Formation of a cold abscess with softened central region

- Stage 4 - Presence of collar-stud abscess

- Stage 5 - Sinus tract formation
Essential features of a tuberculous sinus are bluish, thin, undermined edges with little clear exudates [10]. Three essential features of tuberculous lymphadenitis are multiple, matted caseating lymph nodes. Often, the LD is bilateral and noncontiguous [11]

The recent upsurge in HIV coinfection has challenged the diagnosis and management of TB and of associated LD.

\section{DIAGNOSIS}

In patients presenting with chronic lymph node enlargement, $\mathrm{TB}$ should be given the first differential diagnosis [12].

Regardless of recent advances in diagnostic laboratory skills mycobacterial cervical lymphadenitis still remains a diagnostic dilemma for many clinicians. It is mandatory to differentiate between tuberculous and nontuberculous mycobacterium cervical lymphadenitis as the management strategies varies for the two entities [13].

Table 1 show tuberculous lymphadenitis and nontuberculous lymphadenitis: Differentiating features [12].

1. Age more than 40-50 years. TB lymphadenitis has a predilection for younger age group (20-40 years)

2. Lymphadenitis of the supraclavicular region may be indicative of malignancy

3. No previous history of TB exposure

4. A questionable repeat Bine needle aspiration cytology (FNAC).

5. Nonreactive montoux test

6. Persistance of clinical symptoms and LD after starting anti-TB therapy (ATT).

Most cases of nonresistant TB LD shows lymph node regression within 2-4 months after initiation of ATT. Lack of resolution after 2 months of starting therapy should highlight the resistant TB or non-TB causes.

Diagnostic aids for tuberculous lymphadenitis can be broadly divided into:

- Primary diagnostic aids

- Ancillary diagnostic aids. 


\section{PRIMARY DIAGNOSTIC AIDS}

Culture or polymerase chain reaction (PCR) is essential aids for a conclusive diagnosis of tuberculous lymphadenitis [14].

\section{Culture}

Mycobacterial culture has been the diagnostic choice for tuberculous lymphadenitis. Culture results may be achieved by the use of various media such as Lowenstein Jenson, Middlebrook, BACTEC TB. Major drawback with the use of culture is its slow growth. (requires at least 4 weeks for mycobacterial growth). Micro colony detection on solid media, septicheck acid fast bacilli (AFB) method, microscopic observation of broth culture, BACTEC 460 radiometric system, BACTEC MGIT 960 system, MB/BacT system and ESP II culture system are some of the recent rapid methods used [15] (Fig. 1).

\section{Smears}

Ziehl-Nelson (ZN) staining - ZN staining is an essential diagnostic tool for the identification of AFB and the evaluation of treatment outcome in TB [16]. Bright red rods against blue, green or yellow background are characteristic of AFB [3] (Fig. 2). Major limitations of ZN staining include delayed results, low sensitivity, and use of oil immersion.

Auramine fluorescence - bright rods against dark background are seen with the use of fluorescent microscope [7]. Fluorescent microscopy provides results in a less time and using lower magnifications. Auraminerhodamine or Papanicolaou staining using fluorescent microscope has been suggested to be advanced aid to ZN staining [17,18] (Fig. 3).

\section{FNAC}

FNAC is almost safe, cost-effective and conclusive procedure [19] It serves as a substitute to excision biopsy for lymph nodes and is an easy procedure for sample collection for cytomorphological and

Table 1: Differences between tuberculous and non tuberculous lymphadenitis

\begin{tabular}{|c|c|c|}
\hline & $\begin{array}{l}\text { Tuberculous } \\
\text { lymphadenitis }\end{array}$ & $\begin{array}{l}\text { Non tuberculous } \\
\text { lymphadenitis }\end{array}$ \\
\hline Age range (years) & $20-40$ & $40-60$ \\
\hline Birth country & TB endemic & Non TB endemic \\
\hline Location & Cervical & Cervicofacial \\
\hline Pulmonary disease & Common & Absent \\
\hline Tuberculin skin test & Positive & Occasionally positive \\
\hline FNAC & $\begin{array}{l}\text { FNAC is } \\
\text { conclusive }\end{array}$ & A questionable repeat FNAC \\
\hline Malignancy & Not indicative & $\begin{array}{l}\text { Supraclavicular } \\
\text { lymphadenitis is indicative } \\
\text { of malignancy }\end{array}$ \\
\hline Prognosis & $\begin{array}{l}\text { Cessation } \\
\text { of clinical } \\
\text { symptoms } \\
\text { and LD After } \\
\text { Starting } \\
\text { anti-TB therapy }\end{array}$ & $\begin{array}{l}\text { Persistance of clinical } \\
\text { symptoms and LD after } \\
\text { starting anti-TB therapy }\end{array}$ \\
\hline
\end{tabular}

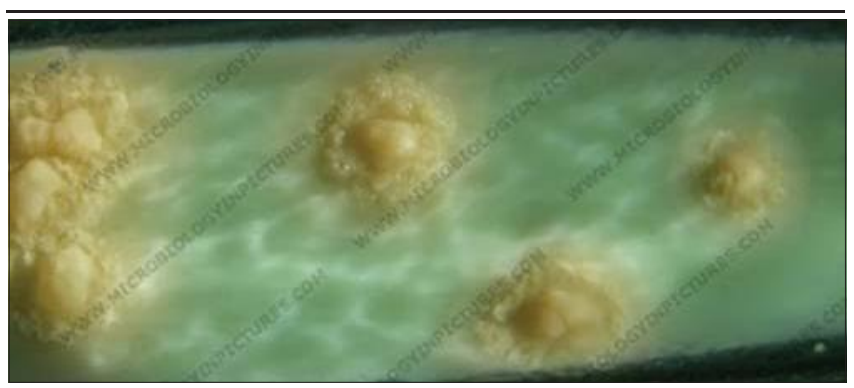

Fig. 1: Lowenstein Jenson Culture medium showing Mycobacterium tuberculosis growth bacteriological examination [20]. FNA cytology has a sensitivity and specificity of $88 \%$ and $96 \%$, respectively, in case of tuberculous lymphadenitis diagnosis [21]. The diagnostic accuracy of mycobacterial cervical lymphadenitis may be greatly enhanced when FNAC is used along with culture or a mantoux test [22-24] (Fig. 4).

Excisional biopsy and histopathology The literature has classically supported excisional biopsyas the definitive diagnostic procedure for diagnosis of nodal TB $[25,26]$. Caseating granulomas with giant cells (langhans and foreign body giant cells) are characteristic for TB. Limitations associated with histopathology are (a) invasive procedure (b) lack of facilities in peripheral health-care centers incisional biopsy is associated with sinus tract and fistula formation, and therefore, is contraindicated [27]. Presently, this technique has been largely replaced by FNAC and histopathology is only reserved for patients with negative FNA despite high clinical suspicion (Fig. 5)

\section{PCR}

The PCR has emerged as a significant aid in the diagnosis of Mycobacterium TB in developed countries [28]. Its sensitivity ranges between $43 \%$ and $84 \%$, and its specificity between $75 \%$ and $100 \%[29,30]$. PCR is the test of choice in smear and culture negative cases [31]. PCR outweighs the conventional diagnostic methods because it is highly sensitive, results can be obtained in few hours, provides distinction between Mycobacterium TB complex and mycobacterial species other than $\mathrm{TB}$, and identifies drug resistance gene mutations

$[32,33]$.

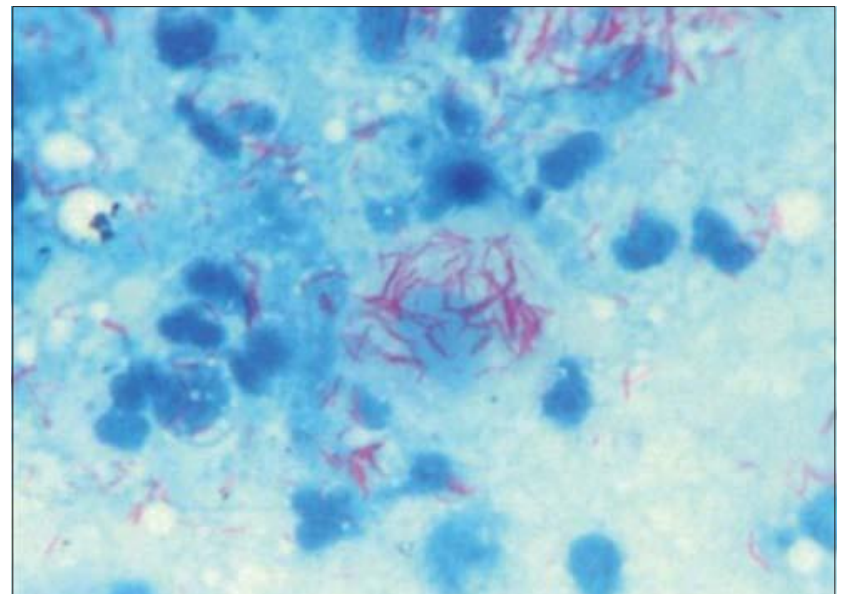

Fig. 2: Zieht-Nelson staining showing bright red rods against blue background

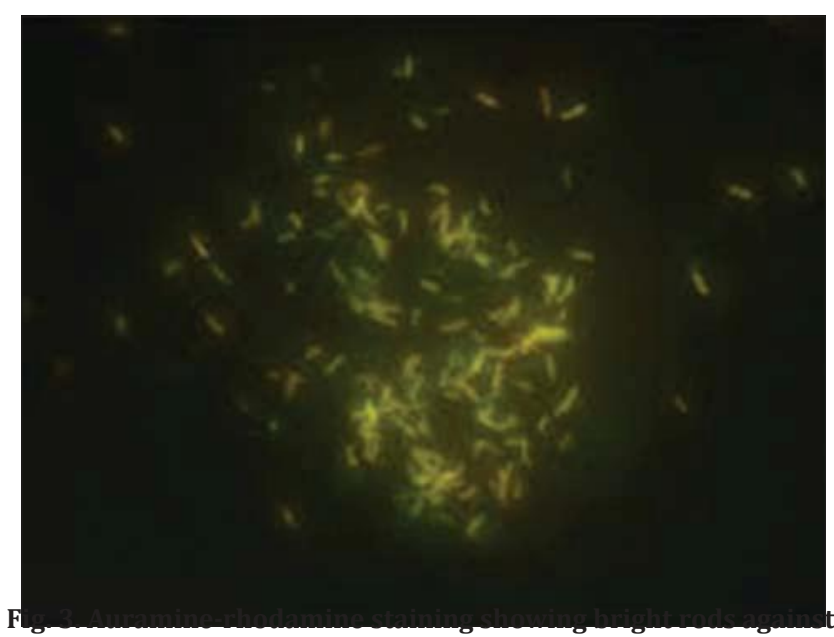

dark background 


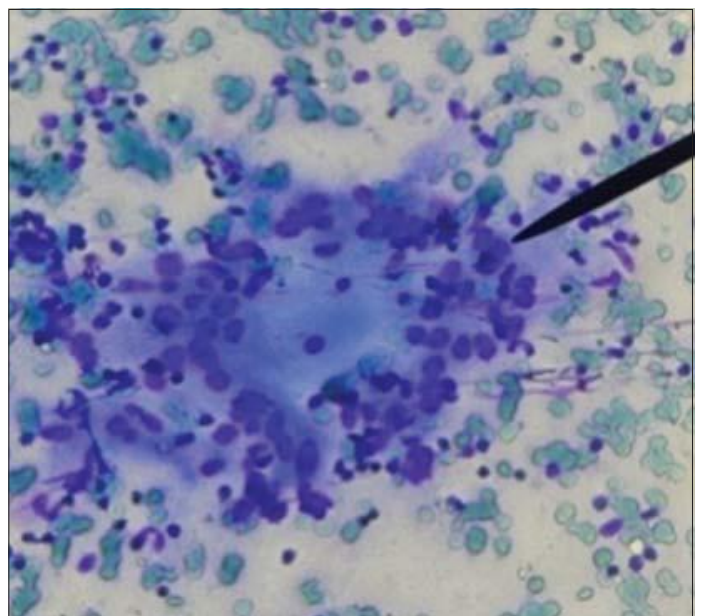

Fig. 4: Fine needle aspiration cytology showing cellular aspirate with giant cells and necrotic debris

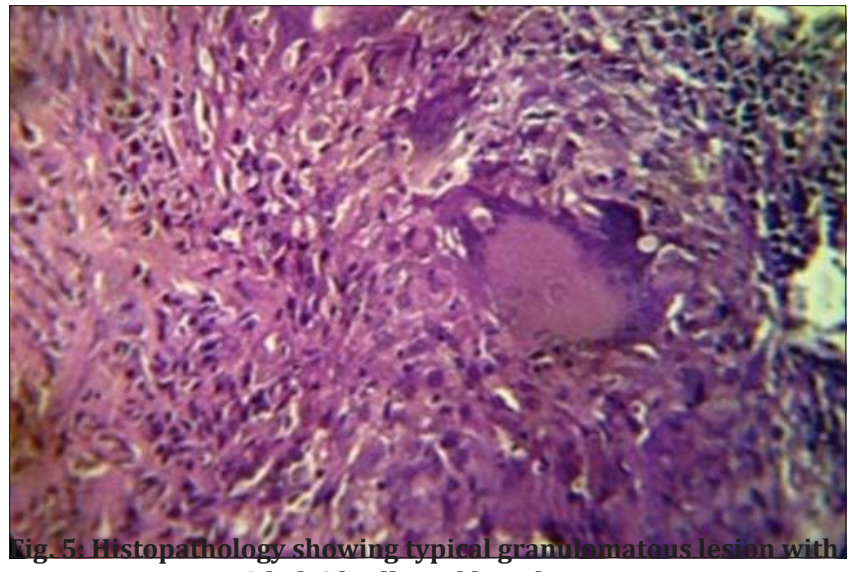

epitheloid cells and lymphocytes

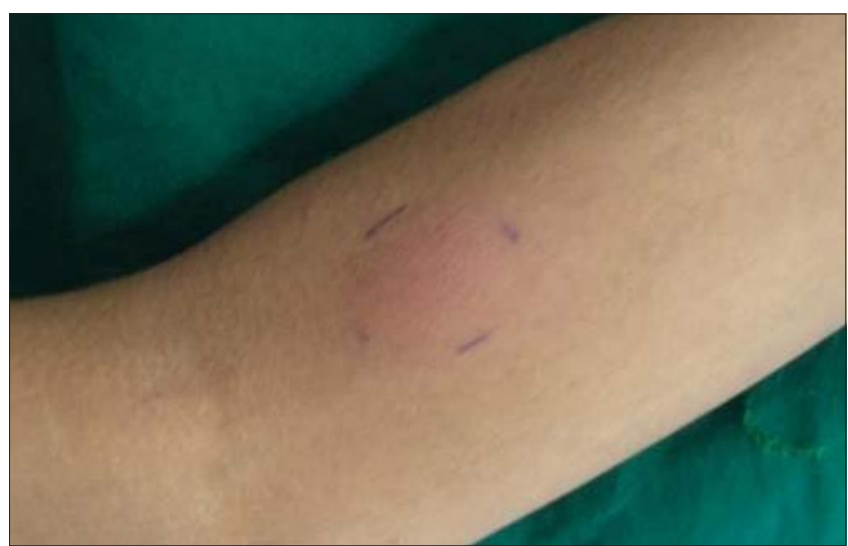

Fig. 6: Induration on the forearm, showing positive tuberculin test

\section{ANCILLARY DIAGNOSTIC TESTS}

\section{Tuberculin skin test/mantoux test (TST)}

It is an intradermal test that demonstrates a delayed type hypersensitivity reaction against mycobacterial antigen. In general, a protein purified derivative is used as the reagent.
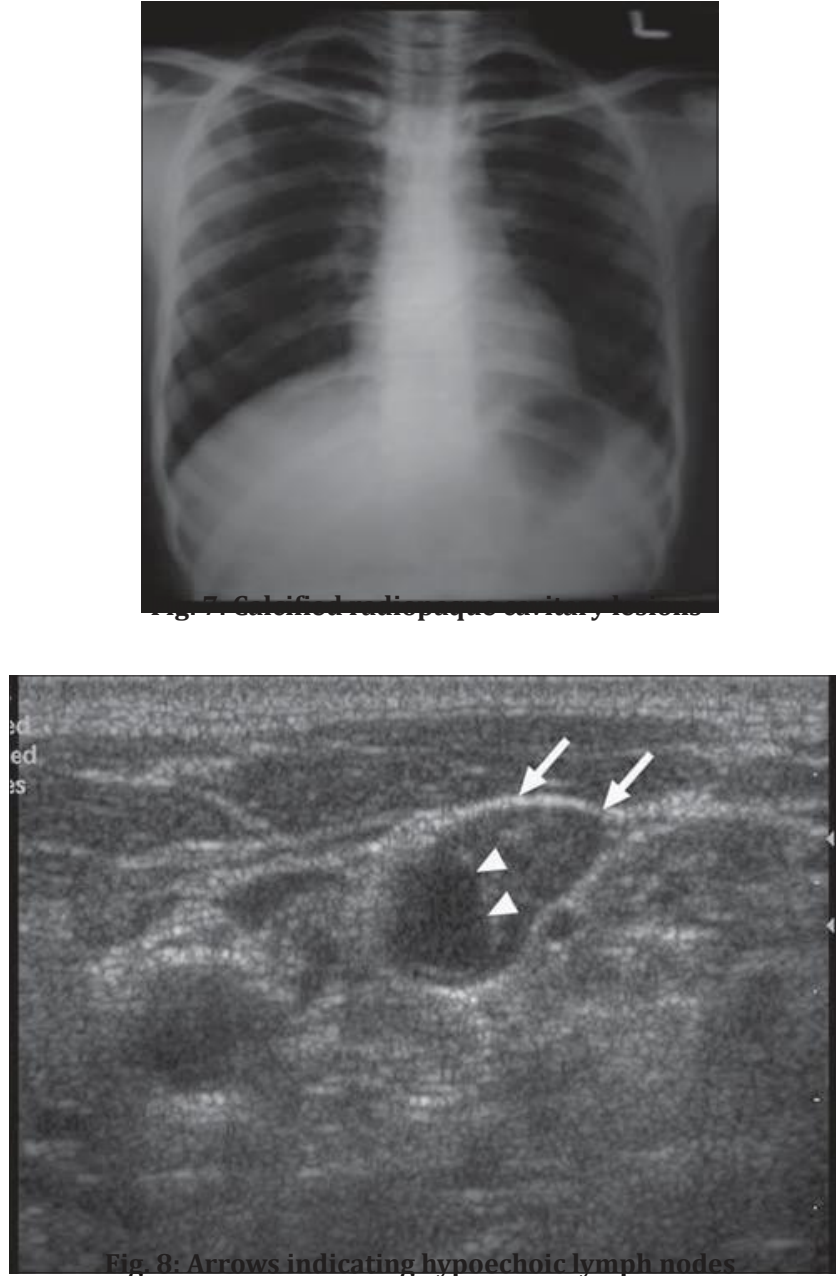

The positive test can be detected after 2-10 weeks of mycobacterial inoculation.

False positive TST reactions may occur in individuals with previous BCG vaccination or nontuberculous mycobacterial infections [31]. Interferon- $\gamma$ release assays have greater specificity than the TST as they do not show false positive reactions with bacille Calmette-Guérin (BCG) or nontuberculous mycobacteria other than Mycobacterium marinum, Mycobacterium kansasii, and Mycobacterium szulgai [34] (Fig. 6).

\section{Chest radiographs}

Chest radiographs may reveal multiple areas of radiolucency (darkened areas), cavities, infiltrates or consolidation in a TB patient [7]. About $10-40 \%$ of the patients show positive chest radiograph findings [13] (Fig. 7).

\section{Ultrasound}

Ultrasound has been used as a rapid, noninvasive, economical, repeatable, and easily available aid for the assessment of cervical lymph nodes. The cytological and pathological details (by the use of ultrasonography guided FNAC and cervical node biopsy) significantly enhances the diagnostic precision of ultrasound [35]. Neck ultrasound reveals increased edema in the surrounding soft-tissue, homogeneity, matting, intranodal cystic necrosis, and posterior enhancement, along with the appearance of lymph node metastases

[13]

(Fig.

8). 
Computed tomography (CT) and magnetic resonance imaging (MRI)

Exact location and extent of the infection may be demonstrated by CT and MRI. Initially, the nodes appear as enlarged, well-defined lymph nodes with homogenous contrast enhancement. Advanced cases show matting with central low attenuation on CT and iso- or hypointense on T2-weighted imaging [36].

\section{CONCLUSION}

Unfortunately, despite several diagnostic aids, TB still remains as one of the leading cause of death from a single infectious organism. About $32 \%$ of the global population is infected with TB and an estimated 2 million people die annually from this treatable disease [37]. The launch of the directly observed treatment short-course strategy by the World Health Organization was expected to substantially curb the incidence of TB [38]. However, an increase in the incidence of mycobacterium TB strain resistance or reduced responsiveness to the first line of anti-TB drugs is a major contributory factor to the current spike in the incidence of this epidemic worldwide [39]. Multidrug-resistant tuberculosis (MDR-TB) is defined as bacilli resistant to at least two first-line agents, isoniazid and rifampin. Drug-resistant tuberculosis developed due to improper previous treatment or interruption of tuberculosis treatment. Treatment of multidrug-resistant TB (MDR-TB) is more than 100 times as costly as treatment of drug susceptible TB, difficult, less effective, requiring intensive care management for its prolonged (18-24 months) and more toxic treatment course [40]. A confirmed diagnosis and differential diagnosis usually needs a high level of expertise and application of a variety of diagnostic modalities. Ideally, diagnosis and treatment should be based on the location of the disease and a thorough clinical evaluation depending on the individual. Treatment with antituberculous medication is essential. Surgery may be required in selected cases.

\section{REFERENCES}

1. Santiago RA, Gueiros LA, Porter SR, Gomes VB, Ferrer I, Leão JC. Prevalence of oral lesions in Brazilian patients with tuberculosis. Indian J Dent Res 2013;24(2):245-8.

2. Kakisi OK, Kechagia AS, Kakisis IK, Rafailidis PI, Falagas ME. Tuberculosis of the oral cavity: A systematic review. Eur J Oral Sci 2010;118(2):103-9.

3. Hasan S, Khan MA. Tuberculosis - A Common Disease with Uncommon Oral Features Report of Two Cases with a Detailed Review of Literature. Proceedings of the World Medical Conference; 2011. p. 156-66.

4. Dandapat MC, Mishra BM, Dash SP, Kar PK. Peripheral lymph node tuberculosis: A review of 80 cases. Br J Surg 1990;77(8):911-2.

5. Shubha AB, Sapna H, Dinesh RB. Tuberculous lymphadenitis presenting a diagnostic dilemma - A case report. Int J Dent Clin 2010;2:48-52.

6. Golden MP, Vikram HR. Extrapulmonary tuberculosis: An overview. Am Fam Physician 2005;72(9):1761-8.

7. Hasan S, Elangovan S. What's your diagnosis? Multiple Jaw Masses. J Dent 2013;3(1):343-51

8. Gupta PR. Difficulties in managing lymph node tuberculosis. Lung India 2004;24:50-3.

9. Jones PG, Campbell PE. Tuberculous lymphadenitis in childhood: The significance of anonymous mycobacteria. Br J Surg 1962;50:302-14.

10. Mohapatra PR, Janmeja AK. Tuberculous lymphadenitis. J Assoc Physicians India 2009;57:585-90.

11. Kent DC. Tuberculous lymphadenitis: Not a localized disease process. Am J Med Sci 1967;254(6):866-74.

12. Thakkar K, Ghaisas SM, Singh M. Lymphadenopathy: Differentiation between tuberculosis and other non-tuberculosis causes like follicular lymphoma. Front Public Health 2016;4:31.

13. Bayazit YA, Bayazit N, Namiduru M. Mycobacterial cervical lymphadenitis. ORL J Otorhinolaryngol Relat Spec 2004;66(5):275-80.

14. Fontanilla JM, Barnes A, von Reyn CF. Current diagnosis and management of peripheral tuberculous lymphadenitis. Clin Infect Dis 2011;53(6):555-62.

15. Indian Council of Medical Research. What is new in the diagnosis of tuberculosis? Part I: Techniques for diagnosis of tuberculosis. ICMR Bull 2002;32(8):69-76.

16. Annam V, Kulkarni MH, Puranik RB. Comparison of the modified fluorescent method and conventional Ziehl-Neelsen method in the detection of acidfast bacilli in lymphnode aspirates. Cytojournal 2009;6(1):13.

17. Jain A, Bhargava A, Agarwal SK. A comparative study of two commonly used staining techniques for acid fast bacilli in clinical specimens. Indian J Tuberc 2002;49:161-2.

18. Wright CA, van Zyl Y, Burgess SM, Blumberg L, Leiman G. Mycobacterial autofluorescence in papanicolaou-stained lymph node aspirates: A glimmer in the dark? Diagn Cytopathol 2004;30(4):257-60.

19. Chaturvedi NK, Singh JP, Amita D. Fine needle aspiration cytology in the diagnosis of tuberculous lymphadenitis. Indian J Pathol Microb 1989;32(2):101-4

20. Ergete W, Bekele A. Acid fast bacilli in aspiration smears from tuberculous patients. Ethiop J Health Dev 2000;14(1):99-104.

21. Chao SS, Loh KS, Tan KK, Chong SM. Tuberculous and nontuberculous cervical lymphadenitis: A clinical review. Otolaryngol Head Neck Surg 2002;126:176-9.

22. Lau SK, Wei WI, Kwan S, Yew WW. Combined use of fine-needle aspiration cytologic examination and tuberculin skin test in the diagnosis of cervical tuberculous lymphadenitis. A prospective study. Arch Otolaryngol Head Neck Surg 1991;117:87-90.

23. Tunkel DE. Surgery for cervicofacial nontuberculous mycobacterial adenitis in children: An update. Arch Otolaryngol Head Neck Surg 1999;125:1109-13

24. Ellison E, Lapuerta P, Martin SE. Fine needle aspiration diagnosis of mycobacterial lymphadenitis. Sensitivity and predictive value in the United States. Acta Cytol 1999;43:153-7.

25. Artenstein AW, Kim JH, Williams WJ, Chung RC. Isolated peripheral tuberculous lymphadenitis in adults: Current clinical and diagnostic issues. Clin Infect Dis 1995;20:876-82.

26. Lee KC, Tami TA, Lalwani AK, Schecter G. Contemporary management of cervical tuberculosis. Laryngoscope 1992;102(1):60-4.

27. Cantrell RW, Jensen JH, Reid D. Diagnosis and management of tuberculous cervical adenitis. Arch Otolaryngol 1975;101(1):53-7.

28. Brown TJ, Power EG, French GL. Evaluation of three commercial detection systems for Mycobacterium tuberculosis where clinical diagnosis is difficult. J Clin Pathol 1999;52(3):193-7.

29. Manitchotpisit B, Kunachak S, Kulapraditharom B, Sura T. Combined use of fine needle aspiration cytology and polymerase chain reaction in the diagnosis of cer vical tuberculous lymphadenitis. J Med Assoc Thai 1999;82:363-8.

30. Hirunwiwatkul P, Tumwasorn S, Chantranuwat C, Sirichai U. A comparative study of diagnostic tests for tuberculous lymphadenitis: Polymerase chain reaction vs histopathology and clinical diagnosis. J Med Assoc Thai 2002;85(3):320-6.

31. Kwon KS, Oh CK, Jang HS, Lee CW, Jun ES. Detection of mycobacterial DNA in cervical granulomatous lymphadenopathy from formalin-fixed, paraffin-embedded tissue by PCR. J Dermatol 2000;27(6):355-60.

32. Piatek AS, Tyagi S, Pol AC, Telenti A, Miller LP, Kramer FR, et al. Molecular beacon sequence analysis for detecting drug resistance in Mycobacterium tuberculosis. Nat Biotechnol 1998;16(4):359-63.

33. Richeldi L, Barnini S, Saltini C. Molecular diagnosis of tuberculosis. Eur Respir J Suppl 1995;20:689s-700.

34. von Reyn CF, Williams DE, Horsburgh CR Jr, Jaeger AS, Marsh BJ, Haslov K, et al. Dual skin testing with Mycobacterium avium sensitin and purified protein derivative to discriminate pulmonary disease due to M. avium complex from pulmonary disease due to Mycobacterium tuberculosis. J Infect Dis 1998;177(3):730-6.

35. Pai M, Riley LW, Colford JM Jr. Interferon-gamma assays in the immunodiagnosis of tuberculosis: A systematic review. Lancet Infect Dis 2004;4(12):761-76.

36. Khanna R, Sharma AD, Khanna S, Kumar M, Shukla RC. Usefulness of ultrasonography for the evaluation of cervical lymphadenopathy. World J Surg Oncol 2011:9:29.

37. Vaid S, Lee YY, Rawat S, Luthra A, Shah D, Ahuja AT. Tuberculosis in the head and neck - A forgotten differential diagnosis. Clin Radiol 2010;65(1):73-81

38. Saeed S, Hasan S. Tuberculosis: A public health challenge. Brief overview of literature. Int Res J Pharm 2016;7(1):1-4

39. Sajith M, Thomas A, Kothia JJ, Chandrakar B, Pawar A, Bargaji MD. Cost of therapy incurred for tuberculosis patients receiving Directly Observed Therapy (DOT). Int J Pharm Pharm Sci 2015;7(10):141-4.

40. Kurniawati F, Sulaiman SA, Gillani SW. Study on drug-resistant tuberculosis and tuberculosis treatment on patients with drug resistant tuberculosis in chest clinic outpatient department. Int J Pharm Pharm Sci 2012;4(2):733-7. 